\title{
ALMOST HERMITIAN HOMOGENEOUS STRUCTURES
}

\author{
by ELSA ABBENA and SERGIO GARBIERO*
}

(Received 13th November 1986)

\section{Introduction}

Let $(M, g, J)$ be an almost Hermitian manifold. More precisely, $M$ is a $\mathscr{C}^{\infty}$ differentiable manifold of dimension $2 n, J$ is an almost complex structure on $M$, i.e. it is a tensor field of type $(1,1)$ such that

$$
J^{2}(X)=-X,
$$

for any $X \in \mathfrak{X}(M),\left(\mathfrak{X}(M)\right.$ is the Lie algebra of $\mathscr{C}^{\infty}$ vector fields on $\left.M\right)$, and $g$ is a Riemannian metric compatible with $J$, i.e.

$$
g(J X, J Y)=g(X, Y), \quad X, Y \in \mathfrak{X}(M) .
$$

$(M, g, J)$ is a homogeneous almost Hermitian manifold if there exists a transitive and effective Lie group $G$ of almost complex isometries acting on $M$. In other words, there is a $\mathscr{C}^{\infty}$ differentiable map

$$
G \times M \rightarrow M, \quad(a, p) \rightarrow L_{a}(p), \quad a \in G, \quad p \in M,
$$

with the following properties

(i) $L_{a b}(p)=L_{a} \cdot L_{b}(p), \quad a, b \in G, p \in M$,

(ii) $L_{a}$ is an isometry of $(M, g)$, for any $a \in G$,

(iii) if $L_{a}(p)=p$ for every $p \in M$, then $a=e(e$ is the unit element of $G$ ),

(iv) for every $p, q \in M$, there is an element $a \in G$ such that $L_{a}(p)=q$,

(v) for all $a \in G, L_{a}$ is an almost complex automorphism of $(M, J)$, i.e.

$$
\left(L_{a}\right)_{*} \cdot J=J \cdot\left(L_{a}\right)_{*}
$$

$\left(\left(L_{a}\right)_{*}\right.$ denotes the differential map of $\left.L_{a}\right)$.

In 1958, Ambrose and Singer gave the following characterization of Riemannian homogeneous manifolds (i.e. Riemannian manifolds admitting an effective and transitive Lie group of isometries).

\footnotetext{
*Work supported by the group G.N.S.A.G.A. of C.N.R. of Italy.
} 
Theorem 1.1 [3]. A connected, simply connected, complete Riemannian manifold $(M, g)$ is homogeneous if and only if there is a tensor field $T$ of type $(1,2)$ on $M$ such that

(i) $\tilde{\nabla} g=0$,

(ii) $\tilde{\nabla} R=0$,

(iii) $\tilde{\nabla} T=0$,

where $\tilde{\nabla}=\nabla-T, \nabla$ is the Levi Civita connection of $M$ and $R$ is the curvature tensor of $\nabla$.

In 1978, Sekigawa found a corresponding theorem for homogeneous almost Hermitian manifolds.

Theorem 1.2 [13]. A connected, simply connected, complete almost Hermitian manifold $(M, g, J)$ is homogeneous if and only if there is a tensor field $T$ of type $(1,2)$ on $M$ which satisfies conditions (i), (ii), (iii) of Theorem 1.1 and (iv) $\tilde{\nabla} J=0$.

A tensor $T$ verifying the conditions of the previous theorem will be called an almost Hermitian homogeneous structure. The aim of this paper is to obtain a classification of almost Hermitian homogeneous structures similar to the classification of Riemannian homogeneous structures given in [16]. In the second section, we decompose the vector space $\mathscr{T}(V)$ of tensors with the same symmetries of the almost Hermitian homogeneous structures in eight subspaces, invariant under the action of the unitary group $U(n)$. Besides, we prove that the decomposition is irreducible by means of the quadratic invariants (see Section 3). Since some subspaces of the decomposition are equivalent in the sense of the representation theory, it follows that there is an infinite number of invariant subspaces of $\mathscr{T}(V)$. As a consequence there are several decompositions. The choice among the different possibilities is motivated by geometrical considerations. For example, from the decomposition of Section 2 we obtain a characterization of homogeneous Kähler manifolds. A second decomposition (see Section 4) is given in order to obtain a connection with the decomposition of $\mathscr{T}(V)$ under the action of the orthogonal group $O(2 n)$ (see [16]). As a consequence, we obtain a classification of naturally reductive homogeneous almost Hermitian manifolds in eight classes (see Section 5). In the last section, some geometrical results and examples are given.

\section{A decomposition of $\mathscr{T}(V)$ into invariant subspaces}

Let $V$ be a real vector space of dimension $2 n$ endowed with a complex structure $J$ and a Hermitian inner product $\langle$, $\rangle$, that is

$$
J^{2}=-I, \quad\langle J X, J Y\rangle=\langle X, Y\rangle,
$$

for any $X, Y \in V$ and $I$ the identity isomorphism of $V$.

In this section, we study the vector space of the tensors which satisfy the symmetries of the almost Hermitian homogeneous structures defined in Theorem 1.2, i.e.

$$
\mathscr{T}(V)=\left\{T \in \stackrel{3}{\otimes} V^{*} / T_{X Y Z}=-T_{X Z Y}\right\},
$$


where $T_{X Y Z}=\left\langle T_{X} Y, Z\right\rangle$, for any $X, Y, Z \in V\left(V^{*}\right.$ is the dual vector space of $\left.V\right) . \mathscr{T}(V)$ has a canonical inner product, induced by $V$, defined in the following way

$$
\langle T, \tilde{T}\rangle=\sum_{i, j, k=1}^{2 n} T_{e_{1} e_{j} e_{k}} \tilde{T}_{e_{i} e_{e k}}
$$

with $T, \tilde{T} \in \mathscr{T}(V)$ and $\left(e_{1}, \ldots, e_{2 n}\right)$ an arbitrary orthonormal basis of $V$. The standard representation $\rho$ of the unitary group $U(n)$ on $V$ gives rise to a representation $\tilde{\rho}$ of $U(n)$ on $\mathscr{T}(V)$ defined by

$$
(\tilde{\rho}(g) T)_{X Y Z}=T_{g^{-1} X_{g}-1 Y_{g}-1 Z}, \quad X, Y, Z \in V, \quad g \in U(n), \quad T \in \mathscr{T}(V) .
$$

It follows immediately that

$$
\langle\tilde{\rho}(g) T, \tilde{\rho}(g) \tilde{T}\rangle=\langle T, \tilde{T}\rangle, \text { for any } T, \tilde{T} \in \mathscr{T}(V),
$$

or, in other words, the map $T \rightarrow \tilde{\rho}(g) T$ is an isometry of $\mathscr{T}(V)$. Therefore, the orthogonal complement of any invariant subspace of $\mathscr{T}(V)$ is still an invariant subspace and the representation $\tilde{\rho}$ is completely reducible. Let us first suppose that $\operatorname{dim} V=2 n$, with $n>2$.

In order to obtain the decomposition into invariant subspaces, we define the endomorphism $\alpha$ of $\mathscr{T}(V)$ as follows

$$
\alpha(T)_{X Y Z}=T_{X J Y J Z}, \quad X, Y, Z \in V .
$$

Since

$$
\alpha^{2}=I \quad \text { and } \quad \alpha(\tilde{\rho}(g) T)=\tilde{\rho}(g)(\alpha T), \quad g \in U(n),
$$

$\alpha$ admits the eigenvalues $+1,-1$ and the eigenspaces

$$
\begin{gathered}
\mathscr{T}(V)_{+}=\left\{T \in \mathscr{T}(V) / T_{X Y Z}=T_{X J Y J Z}, X, Y, Z \in V\right\}, \\
\mathscr{T}(V)_{-}=\left\{T \in \mathscr{T}(V) / T_{X Y Z}=-T_{X J Y J Z}, X, Y, Z \in V\right\},
\end{gathered}
$$

are invariant and mutually orthogonal, because $\alpha$ is a symmetric operator with respect to the inner product in $\mathscr{T}(V)$.

Moreover

$$
\mathscr{T}(V)=\mathscr{T}(V)_{+} \oplus \mathscr{T}(V)_{-}
$$

These subspaces are not irreducible under the action of $U(n)$.

In the next theorem, a decomposition of $\mathscr{T}(V)_{+}$is given.

Theorem 2.1. In the case of $\operatorname{dim} V=2 n$, with $n>2$, we have

$$
\mathscr{T}(V)_{+}=\mathscr{H}_{1} \oplus \mathscr{H}_{2} \oplus \mathscr{H}_{3} \oplus \mathscr{H}_{4},
$$


where

$$
\begin{aligned}
\mathscr{H}_{1}=\left\{T \in \mathscr{T}(V)_{+} / T_{X Y Z}\right. & \left.=\frac{1}{2}\left(T_{Z X Y}+T_{Y Z X}+T_{J Z X J Y}+T_{J Y J Z X}\right), c_{12}(T)=0\right\}, \\
\mathscr{H}_{2}=\left\{T \in \mathscr{T}(V)_{+} / T_{X Y Z}=\right. & \langle X, Y\rangle \psi_{1}(Z)-\langle X, Z\rangle \psi_{1}(Y)+\langle X, J Y\rangle \psi_{1}(J Z) \\
& \left.-\langle X, J Z\rangle \psi_{1}(J Y)-2\langle J Y, Z\rangle \psi_{1}(J X), \psi_{1} \in V^{*}\right\}, \\
\mathscr{H}_{3}=\left\{T \in \mathscr{T}(V)_{+} / T_{X Y Z}\right. & \left.=-\frac{1}{2}\left(T_{Z X Y}+T_{Y Z X}+T_{J Z X J Y}+T_{J Y J Z X}\right), c_{12}(T)=0\right\}, \\
\mathscr{H}_{4}=\left\{T \in \mathscr{T}(V)_{+} / T_{X Y Z}=\right. & \langle X, Y\rangle \psi_{2}(Z)-\langle X, Z\rangle \psi_{2}(Y)+\langle X, J Y\rangle \psi_{2}(J Z) \\
& \left.-\langle X, J Z\rangle \psi_{2}(J Y)+2\langle J Y, Z\rangle \psi_{2}(J X), \psi_{2} \in V^{*}\right\},
\end{aligned}
$$

for any $X, Y, Z \in V . c_{12}$ is defined by

$$
c_{12}(T)(X)=\sum_{i=1}^{2 n} T_{e_{i} e_{i} X}, \quad \text { for any } X \in V,
$$

and $\left(e_{1}, \ldots, e_{2 n}\right)$ an arbitrary orthonormal basis of $V$.

The subspaces $\mathscr{H}_{1}, \mathscr{H}_{2}, \mathscr{H}_{3}, \mathscr{H}_{4}$ are mutually orthogonal and invariant under the action of $U(n)$ on $\mathscr{T}(V)_{+}$.

If $\operatorname{dim} V=2$, then $\mathscr{T}(V)_{+}=\mathscr{H}_{4}$. If $\operatorname{dim} V=4$, then $\mathscr{T}(V)_{+}=\mathscr{H}_{2} \oplus \mathscr{H}_{3} \oplus \mathscr{H}_{4}$.

Proof. Let $\beta$ be the endomorphism of $\mathscr{T}(V)_{+}$given by

$$
\beta(T)_{X Y Z}=\frac{1}{2}\left(T_{Z X Y}+T_{Y Z X}+T_{J Z X J Y}+T_{J Y J Z X}\right), \quad X, Y, Z \in V .
$$

Since $\beta^{2}=I$ and $\beta$ commutes with the action of $U(n), \mathscr{T}(V)_{+}$can be decomposed into the direct sum of two mutually orthogonal and invariant subspaces

$$
\begin{gathered}
\mathscr{P}=\left\{T \in \mathscr{T}(V)_{+} / \beta(T)=+T\right\}, \\
\mathscr{Q}=\left\{T \in \mathscr{T}(V)_{+} / \beta(T)=-T\right\} .
\end{gathered}
$$

Using the trace, one can see that $\mathscr{P}$ and $\mathscr{Q}$ further split in the following way

$$
\begin{gathered}
\mathscr{H}_{1}=\left\{T \in \mathscr{P} / c_{12}(T)=0\right\}, \\
\mathscr{H}_{2}=\mathscr{H}_{1}^{\perp}, \\
\mathscr{H}_{3}=\left\{T \in \mathscr{Q}_{c_{12}}(T)=0\right\}, \\
\mathscr{H}_{4}=\mathscr{H}_{3}^{\perp},
\end{gathered}
$$

where $\mathscr{H}_{1}^{\perp}\left(\mathscr{H}_{3}^{\perp}\right)$ is the orthogonal complement of $\mathscr{H}_{1}\left(\mathscr{H}_{3}\right)$ in $\mathscr{P}(\mathscr{Q})$. 
By an explicit calculation we get the expressions of $\mathscr{H}_{2}$ and $\mathscr{H}_{4}$ and we find

$$
\psi_{1}(X)=\frac{1}{2(n-1)} c_{12}(T)(X), \quad \psi_{2}(X)=\frac{1}{2(n+1)} c_{12}(T)(X), \quad X \in V .
$$

Gray and Hervella [9] decomposed the vector space

$$
\mathscr{W}=\left\{\alpha \in \bigotimes^{3} V^{*} / \alpha(X, Y, Z)=-\alpha(X, Z, Y)=-\alpha(X, J Y, J Z), \quad X, Y, Z \in V\right\},
$$

into four irreducible and invariant (under the action of $U(n)$ ) subspaces. Because $\mathscr{T}(V)_{-}=\mathscr{W}$, the following theorem holds:

Theorem 2.2 [9]. If $\operatorname{dim} V=2 n, n>2$, we have: $\mathscr{T}(V)_{-}=\mathscr{H}_{5} \oplus \mathscr{H}_{6} \oplus \mathscr{H}_{7} \oplus \mathscr{H}_{8}$, where:

$$
\begin{gathered}
\mathscr{H}_{5}=\left\{T \in \mathscr{T}(V)_{-} / T_{X X Z}=0\right\}, \\
\mathscr{H}_{6}=\left\{\left.T \in \mathscr{T}(V)_{-}\right|_{X Y Z} ^{\sigma} T_{X Y Z}=0\right\}, \\
\mathscr{H}_{7}=\left\{T \in \mathscr{T}(V)_{-} / T_{X Y Z}=T_{J X J Y Z}, c_{12}(T)=0\right\}, \\
\mathscr{H}_{8}=\left\{T \in \mathscr{T}(V)_{-} / T_{X Y Z}=\langle X, Y\rangle \psi_{3}(Z)-\langle X, Z\rangle \psi_{3}(Y)-\langle X, J Y\rangle \psi_{3}(J Z)\right. \\
\left.+\langle X, J Z\rangle \psi_{3}(J Y), \psi_{3} \in V^{*}\right\},
\end{gathered}
$$

for any $X, Y, Z \in V$ and $\mathfrak{G}_{X Y Z}$ denotes the cyclic sum with respect to $X, Y, Z$. The subspaces $\mathscr{H}_{5}, \mathscr{H}_{6}, \mathscr{H}_{7}, \mathscr{H}_{8}$ are mutually orthogonal and invariant under the action of $U(n)$ on $\mathscr{T}(V)$. Moreover, we have

$$
\psi_{3}(X)=\frac{1}{2(n-1)} c_{12}(T)(X), \quad X \in V .
$$

If $\operatorname{dim} V=2$ then $\mathscr{T}(V)=\{0\}$. If $\operatorname{dim} V=4$ then $\mathscr{T}(V)_{-}=\mathscr{H}_{6} \oplus \mathscr{H}_{8}$.

Theorem 2.3. Let $\operatorname{dim} V \geqq 6$, then $\mathscr{T}(V)$ is the orthogonal direct sum of the invariant subspaces $\mathscr{H}_{i}, i=1, \ldots, 8$ given by

$$
\begin{aligned}
\mathscr{H}_{1}=\left\{T \in \mathscr{T}(V) / T_{X Y Z}=\right. & \left.\frac{1}{2}\left(T_{Z X Y}+T_{Y Z X}+T_{J Z X J Y}+T_{J Y J Z X}\right), c_{12}(T)=0\right\}, \\
\mathscr{H}_{2}=\left\{T \in \mathscr{T}(V) / T_{X Y Z}=\right. & \langle X, Y\rangle \psi_{1}(Z)-\langle X, Z\rangle \psi_{1}(Y)+\langle X, J Y\rangle \psi_{1}(J Z) \\
& \left.-\langle X, J Z\rangle \psi_{1}(J Y)-2\langle J Y, Z\rangle \psi_{1}(J X), \psi_{1} \in V^{*}\right\}, \\
\mathscr{H}_{3}=\left\{T \in \mathscr{T}(V) / T_{X Y Z}=\right. & \left.-\frac{1}{2}\left(T_{Z X Y}+T_{Y Z X}+T_{J Z X J Y}+T_{J Y J Z X}\right), c_{12}(T)=0\right\},
\end{aligned}
$$




$$
\begin{gathered}
\mathscr{H}_{4}=\left\{T \in \mathscr{T}(V) / T_{X Y Z}=\langle X, Y\rangle \psi_{2}(Z)-\langle X, Z\rangle \psi_{2}(Y)+\langle X, J Y\rangle \psi_{2}(J Z)\right. \\
\left.-\langle X, J Z\rangle \psi_{2}(J Y)+2\langle J Y, Z\rangle \psi_{2}(J X), \psi_{2} \in V^{*}\right\}, \\
\mathscr{H}_{5}=\left\{T \in \mathscr{T}(V) / T_{X Y Z}=-T_{Y X Z}=-T_{X J Y J Z}\right\}, \\
\mathscr{H}_{6}=\left\{T \in \mathscr{T}(V) / \mathfrak{G}_{X Y Z} T_{X Y Z}=0, T_{X Y Z}=-T_{X J Y J Z}\right\}, \\
\mathscr{H}_{7}=\left\{T \in \mathscr{T}(V) / T_{X Y Z}=-T_{X J Y J Z}=T_{J X J Y Z}, c_{12}(T)=0\right\}, \\
\mathscr{H}_{8}=\left\{T \in \mathscr{T}(V) / T_{X Y Z}=\langle X, Y\rangle \psi_{3}(Z)-\langle X, Z\rangle \psi_{3}(Y)+\langle X, J Z\rangle \psi_{3}(J Y)\right. \\
\left.-\langle X, J Y\rangle \psi_{3}(J Z), \psi_{3} \in V^{*}\right\} .
\end{gathered}
$$

If $\operatorname{dim} V=2$, then $\mathscr{T}(V)=\mathscr{H}_{4}$.

If $\operatorname{dim} V=4$, then $\mathscr{T}(V)=\mathscr{H}_{2} \oplus \mathscr{H}_{3} \oplus \mathscr{H}_{4} \oplus \mathscr{H}_{6} \oplus \mathscr{H}_{8}$.

\section{Irreducibility of the decomposition and dimensions of the subspaces}

In order to prove that the decomposition given in Theorem 2.3 is irreducible under the action of $U(n)$, it is necessary to find a set of generators for the vector space of the quadratic invariants of $\mathscr{T}(V)$.

In fact the following theorem holds:

Theorem 3.1. A subspace of $\otimes V^{*}$, invariant under the action of $U(n)$, is irreducible if and only if the vector space of its quadratic invariants has dimension one (see for example [19]).

The quadratic invariants of $\mathscr{T}(V)$ can be obtained, by restriction, from those of ()$^{3} V^{*}$. We have:

Theorem 3.2. The vector space of the quadratic invariants of $\mathscr{T}(V)$ is generated by

$$
\begin{gathered}
i_{1}(T)=\|T\|^{2}=\sum_{i, j, k=1}^{2 n} T_{e_{i} e_{j} e_{k}}^{2}, \\
i_{2}(T)=\left\|c_{12}(T)\right\|^{2}=\sum_{i, j, k=1}^{2 n} T_{e_{i} e_{i} e_{k}} T_{e_{j} e_{e} e_{k}}, \\
i_{3}(T)=\sum_{i, j, k=1}^{2 n} T_{e_{i} e_{j} e_{k}} T_{e e_{i} e_{k}}, \\
i_{4}(T)=\sum_{i, j, k=1}^{2 n} T_{e_{i} e_{e j} e_{j}} T_{e j e_{k} J e_{k}},
\end{gathered}
$$




$$
\begin{aligned}
& i_{S}(T)=\sum_{i, j, k=1}^{2 n} T_{e_{i} e_{i} e_{j}} T_{\text {Jekekej }}, \\
& i_{6}(T)=\sum_{i, j, k=1}^{2 n} T_{e_{i} e_{j} e_{k}} T_{J e j e_{i} e_{k}}, \\
& i_{7}(T) \sum_{i, j, k=1}^{2 n} T_{e_{i} e_{j} e_{k}} T_{J e_{i} J e_{j} e_{k}} \\
& i_{8}(T)=\sum_{i, j, k=1}^{2 n} T_{e_{i} e_{j} e_{k}} T_{e_{i} J e_{j} J e_{k}} \\
& i_{9}(T)=\sum_{i, j, k=1}^{2 n} T_{e_{i} e_{j} e_{k}} T_{J e_{k} J e_{i} e_{j}} \\
& i_{10}(T)=\sum_{i, j, k=1}^{2 n} T_{e_{i} e_{j} e_{k}} T_{e_{k} J e_{j} J e_{j}}, \\
& i_{11}(T)=\sum_{i, j, k=1}^{2 n} T_{e_{i} e_{i} e_{j}} T_{J e_{j} J e_{k} e_{k}}, \\
& i_{12}(T)=\sum_{i, j, k=1}^{2 n} T_{e_{i} e_{i} e_{j}} T_{J e_{k} e_{k} J e_{j}}, \\
& i_{13}(T)=\sum_{i, j, k=1}^{2 n} T_{J e_{i} e_{j} J e_{j}} T_{e j J e_{k} e_{k}}, \\
& i_{14}(T)=\sum_{i, j, k=1}^{2 n} T_{J e_{i} e_{i} e_{j}} T_{e, J e_{k} e_{k}}, \\
& i_{15}(T)=\sum_{i, j, k=1}^{2 n} T_{e j} J e_{i} e_{i} T_{e j J e_{k} e_{k}}, \\
& i_{16}(T)=\left\|\bar{c}_{12}(T)\right\|^{2}=\sum_{i, j, k=1}^{2 n} T_{J e_{l} e_{i} e_{j}} T_{J e_{k} e_{k} e_{j}}
\end{aligned}
$$

where $\left(e_{1}, \ldots, e_{2 n}\right)$ is an arbitrary orthonormal basis of $V$ and $T \in \mathscr{T}(V)$.

Proof. From [12], we know that each quadratic invariant of $\bigotimes^{3} V^{*}$ can be written as

$$
\mathscr{P}(T)=\sum_{i_{p}, j_{q}=1}^{2 n} T_{e_{e_{1}} e_{i_{2}} e_{i_{3}}} T_{e_{e_{1}}, e_{2} e_{2} e_{3}} \not\left(e_{i_{1}}, e_{i_{2}}, e_{i_{3}}, e_{j_{1}}, e_{j_{2}}, e_{j_{3}}\right),
$$

where $T \in \mathscr{T}(V)$ and $\not h$ is a linear invariant of $\otimes^{6} V^{*}$, i.e. $\not h$ is a linear combination of elements of the following type 


$$
F_{\alpha_{1} \alpha_{2} \alpha_{3}}^{\sigma}\left(X_{1}, \ldots, X_{6}\right)=\Omega_{\alpha_{1}}\left(X_{\sigma(1)}, X_{\sigma(2)}\right) \Omega_{\alpha_{2}}\left(X_{\sigma(3)}, X_{\sigma(4)}\right) \Omega_{\alpha_{3}}\left(X_{\sigma(5)}, X_{\sigma(6)}\right)
$$

for $X_{i} \in V, i=1, \ldots, 6 ; \sigma$ is a permutation of $(1, \ldots, 6), \alpha_{i}=0,1$ and

$$
\Omega_{0}(X, Y)=\langle X, Y\rangle, \quad \Omega_{1}(X, Y)=\langle X, J Y\rangle
$$

A straightforward, but lengthy, computation gives the above result. has

By checking directly that Theorem 3.1 is satisfied for any subspace $\mathscr{H}_{i}, i=1, \ldots, 8$, one

Theorem 3.3. The decomposition of $\mathscr{T}(V)$ given in Theorem 2.3 is irreducible under the action of $U(n)$.

The dimensions of the irreducible subspace are the following (see also [9]):

Theorem 3.4. If $\operatorname{dim} V=2 n, n>2$, then

$$
\begin{gathered}
\operatorname{dim} \mathscr{H}_{1}=\operatorname{dim} \mathscr{H}_{7}=n(n+1)(n-2), \\
\operatorname{dim} \mathscr{H}_{2}=\operatorname{dim} \mathscr{H}_{4}=\operatorname{dim} \mathscr{H}_{8}=2 n, \\
\operatorname{dim} \mathscr{H}_{3}=n(n-1)(n+2), \\
\operatorname{dim} \mathscr{H}_{5}=\frac{1}{3} n(n-1)(n-2), \\
\operatorname{dim} \mathscr{H}_{6}=\frac{2}{3} n(n-1)(n+1) .
\end{gathered}
$$

If $\operatorname{dim} V=2,4$ we get the particular cases described in Theorem 2.3.

Proof. In the case of $\operatorname{dim} V=2 n, n>2$, the dimensions of $\mathscr{H}_{5}, \mathscr{H}_{6}, \mathscr{H}_{7}, \mathscr{H}_{8}$ can be found in [9, remark on page 39]. Obviously, $\mathscr{H}_{2}$ and $\mathscr{H}_{4}$ have dimension $2 n$. Hence it follows that

$$
\operatorname{dim} \mathscr{H}_{1} \oplus \mathscr{H}_{3}=\operatorname{dim} \mathscr{T}(V)-\operatorname{dim}\left(\mathscr{H}_{2} \oplus \mathscr{H}_{4} \oplus \mathscr{T}(V)_{-}\right)=2 n\left(n^{2}-2\right)
$$

Writing down explicitly the conditions which must be satisfied by the components of a tensor $T \in \mathscr{H}_{1}$, the dimension of $\mathscr{H}_{1}$ follows from a long, but elementary, calculation.

\section{Another decomposition of $\mathscr{T}(V)$}

In this section we give a different decomposition of $\mathscr{T}(V)$, with respect to the action of $U(n)$. This one will be strictly related to the decomposition found by Tricerri and Vanhecke if $\mathscr{T}(V)$ is acted on by the orthogonal group $O(2 n)$. In fact, according to 
Theorem 3.1 of [16], we have that

$$
\mathscr{T}(V)=\mathscr{T}_{1}(V) \oplus \mathscr{T}_{2}(V) \oplus \mathscr{T}_{3}(V)
$$

where

$$
\begin{gathered}
\mathscr{T}_{1}(V)=\left\{T \in \mathscr{T}(V) / T_{X Y Z}=\langle X, Y\rangle \phi(Z)-\langle X, Z\rangle \phi(Y), \phi \in V^{*}\right\}, \\
\mathscr{T}_{2}(V)=\left\{T \in \mathscr{T}(V) / \mathfrak{G}_{X Y Z} T_{X Y Z}=0, c_{12}(T)=0\right\}, \\
\mathscr{T}_{3}(V)=\left\{T \in \mathscr{T}(V) / T_{X Y Z}+T_{Y X Z}=0\right\},
\end{gathered}
$$

with $X, Y, Z \in V$ and $\left(_{X Y Z}\right.$ denotes the cyclic sum with respect to $X, Y, Z$. Moreover, $\mathscr{T}_{1}(V), \mathscr{T}_{2}(V), \mathscr{T}_{3}(V)$ are irreducible and invariant with respect to $O(2 n)$. They are invariant under the action of $U(n)$, too.

On the other hand, Gray and Hervella [9] gave a decomposition of the vector space $\mathscr{W}$ under the action of $U(n)$. Here, $\mathscr{W}$ denotes the vector space of the tensors with the same symmetries as $\nabla F$, where $F$ is the Kähler form of an almost Hermitian manifold and $\nabla$ is the Levi Civita connection of the Hermitian metric. More precisely, if

$$
\left.\mathscr{W}=\{\alpha \in \otimes)^{3} V^{*} / \alpha(X, Y, Z)=-\alpha(X, Z, Y)=-\alpha(X, J Y, J Z)\right\},
$$

we have

$$
\mathscr{W}=\mathscr{W}_{1} \oplus \mathscr{W}_{2} \oplus \mathscr{W}_{3} \oplus \mathscr{W}_{4}
$$

where

$$
\begin{gathered}
\mathscr{W}_{1}=\{\alpha \in \mathscr{W} / \alpha(X, X, Z)=0\}, \\
\mathscr{W}_{2}=\{\alpha \in \mathscr{W} / \alpha(X, Y, Z)+\alpha(Z, X, Y)+\alpha(Y, Z, X)=0\}, \\
\mathscr{W}_{3}=\left\{\alpha \in \mathscr{W} / \alpha(X, Y, Z)-\alpha(J X, J Y, Z)=0, c_{12}(\alpha)=0\right\}, \\
\mathscr{W}_{4}=\left\{\alpha \in \mathscr{W} / \alpha(X, Y, Z)=\langle X, Y\rangle \eta(Z)-\langle X, Z\rangle_{\eta}(Y)-\langle X, J Y\rangle_{\eta}(J Z)\right. \\
\left.+\langle X, J Z\rangle \eta(J Y), \eta \in V^{*}\right\},
\end{gathered}
$$

for any $X, Y, Z \in V$. The subspaces $\mathscr{W}_{i}, i=1, \ldots, 4$, are irreducible and invariant under the action of $U(n)$.

Let $(M, g, J)$ be a homogeneous almost Hermitian manifold. From condition (iv) of Theorem 1.2, we deduce the following relation between the tensor $T$ and $\nabla F$

$$
\left(\nabla_{X} F\right)(Y, Z)=T_{X J Y Z}+T_{X Y J Z} \quad X, Y, Z \in \mathfrak{X}(M) .
$$


This suggests we consider the homomorphism

$$
\psi: \mathscr{T}(V) \rightarrow \mathscr{W}, \quad \psi(T)_{X Y Z}=T_{X J Y Z}+T_{X Y J Z}, \quad X, Y, Z \in V .
$$

Obviously, $\psi$ commutes with the action of the unitary group $U(n)$. Let $\psi_{i}, i=1,2,3$, be the restriction of $\psi$ to the subspaces $\mathscr{T}_{i}(V), i=1,2,3$. We have

Theorem 4.1. The homomorphisms

$$
\begin{gathered}
\psi_{1}: \mathscr{T}_{1}(V) \rightarrow \mathscr{W}_{4}, \\
\psi_{3}: \mathscr{T}_{3}(V) \rightarrow \mathscr{W}_{1} \oplus \mathscr{W}_{3} \oplus \mathscr{W}_{4},
\end{gathered}
$$

are isomorphisms and they commute with the action of $U(n)$. then

Proof. It is clear that $\psi_{1}$ is an isomorphism between $\mathscr{T}_{1}(V)$ and $\mathscr{W}_{4}$. If $T \in \mathscr{T}_{3}(V)$,

$$
\psi(T)_{X X Y}-\psi(T)_{J X J X Y}=0, \quad X, Y \in V,
$$

that is $\psi(T) \in \mathscr{W}_{1} \oplus \mathscr{W}_{3} \oplus \mathscr{W}_{4}$ (see [9]). Since $\operatorname{dim} \mathscr{T}_{3}(V)=\operatorname{dim} \mathscr{W}_{1} \oplus \mathscr{W}_{3} \oplus \mathscr{W}_{4}=\left(\begin{array}{c}2 n \\ 3\end{array}\right)$ (see [9] and [16]), it is enough to show that $\psi_{3}$ is injective. But $\operatorname{Ker} \psi_{3}=$ $\left\{T \in \mathscr{T}_{3}(V) / T_{X Y Z}=T_{X J Y J Z}\right\}$ is trivial as can be seen by the following computation. Let $T \in \operatorname{Ker} \psi_{3}$, then from

$$
T_{X Y Z}=-T_{Y X Z}=T_{X J Y J Z}, \quad X, Y, Z \in V,
$$

we obtain

$$
T_{X J Y Z}=T_{J X Y Z}, \quad X, Y, Z \in V .
$$

Since $T$ is a 3 -form on $V$, we finally get

$$
T_{J X Y Z}=T_{X J Y Z}=-T_{X Y J Z}=-T_{J Z X Y}=-T_{Z J X Y}=T_{J X Z Y}=-T_{J X Y Z}, \quad X, Y, Z \in V .
$$

The last part of the theorem is obvious.

Theorem 4.2. The homomorphism

$$
\psi_{2}: \mathscr{T}_{2}(V) \rightarrow \mathscr{W}_{2} \oplus \mathscr{W}_{3} \oplus \mathscr{W}_{4}
$$

is surjective and commutes with the action of $U(n)$. 
Proof. If $T \in \mathscr{T}_{2}(V)$,

$$
\boldsymbol{G}_{X Y Z}\left\{(\psi T)_{X Y Z}-(\psi T)_{J X J Y Z}\right\}=0
$$

i.e. $\psi(T) \in \mathscr{W}_{2} \oplus \mathscr{W}_{3} \oplus \mathscr{W}_{4}$. By definition:

$\operatorname{Ker} \psi_{2}=\left\{T \in \mathscr{T}(V) / \mathfrak{G}_{X Y Z} T_{X Y Z}=0, T_{X Y Z}=T_{X J Y J Z}, c_{12}(T)=0, X, Y, Z \in V\right\}$

Let $\left(e_{1}, \ldots, e_{n}, e_{n+1}=J e_{1}, \ldots, e_{2 n}=J e_{n}\right)$ be an orthonormal basis of $V$. The above conditions imply that $T \in \operatorname{Ker} \psi_{2}$ is completely determined by the relations

$$
T_{e_{i} e_{a} e_{n+b}}=T_{e_{i} e_{b} e_{n+a}}, \quad \sum_{k=1}^{2 n} T_{e_{k} e_{k} e_{i}}=0, \quad i=1, \ldots, 2 n, \quad a, b=1, \ldots, n .
$$

It follows that

$$
\operatorname{dim} \operatorname{Ker} \psi_{2}=\frac{n(n+1)}{2} 2 n-2 n=n(n-1)(n+2)
$$

From [16] and [9], we get

$$
\operatorname{dim} \mathscr{T}_{2}(V)=\frac{8 n(n+1)(n-1)}{3} \text { and } \operatorname{dim} \mathscr{W}_{2} \oplus \mathscr{W}_{3} \oplus \mathscr{W}_{4}=\frac{n(n-1)(5 n+2)}{3}
$$

hence the theorem holds.

From the last two theorems we have:

Theorem 4.3. Let $\operatorname{dim} V=2 n, n>2$, then

$$
\mathscr{T}(V)=\mathscr{T}_{1}(V) \oplus \operatorname{Ker} \psi_{2} \oplus \mathscr{W}_{2}^{\prime} \oplus \mathscr{W}_{3}^{\prime} \oplus \mathscr{W}_{4}^{\prime} \oplus \mathscr{W}_{1}^{\prime \prime} \oplus \mathscr{W}_{3}^{\prime \prime} \oplus \mathscr{W}_{4}^{\prime \prime}
$$

where

$$
\psi_{2}\left(\mathscr{W}_{i}^{\prime}\right)=\mathscr{W}_{i}, \quad i=2,3,4, \quad \psi_{3}\left(\mathscr{W}_{j}^{\prime \prime}\right)=\mathscr{W}_{j}, \quad j=1,3,4
$$

If $\operatorname{dim} V=2$, then $\mathscr{T}(V)=\mathscr{T}_{1}(V)$. If $\operatorname{dim} V=4$, then

$$
\mathscr{T}(V)=\mathscr{T}_{1}(V) \oplus \operatorname{Ker} \psi_{2} \oplus \mathscr{W}_{2}^{\prime} \oplus \mathscr{W}_{4}^{\prime} \oplus \mathbb{W}_{4}^{\prime \prime}
$$

Moreover, all the subspaces are mutually orthogonal, irreducible and invariant under the action of $U(n)$.

Proof. We need only to prove the irreducibility of the decomposition. For this purpose we apply Theorem 3.1 . 
Remarks. (a) Using the above homomorphisms, one can see that, if $\operatorname{dim} V=2 n$, $n>2$, the dimensions of the subspaces are

$$
\begin{gathered}
\operatorname{dim} \mathscr{T}_{1}(V)=\operatorname{dim} \mathscr{W}_{4}^{\prime}=\operatorname{dim} \mathscr{W}_{4}^{\prime \prime}=2 n, \\
\operatorname{dim} \operatorname{Ker} \psi_{2}=n(n-1)(n+2) \\
\operatorname{dim} \mathscr{W}_{1}^{\prime \prime}=\frac{1}{3} n(n-1)(n-2), \\
\operatorname{dim} \mathscr{W}_{2}^{\prime}=\frac{2}{3} n(n+1)(n-1), \\
\operatorname{dim} \mathscr{W}_{3}^{\prime}=\operatorname{dim} \mathscr{W}_{3}^{\prime \prime}=n(n+1)(n-2)
\end{gathered}
$$

(b) The reason why there are several decompositions of the space $\mathscr{T}(V)$ under the action of $U(n)$ is due to the fact that there exist equivalent irreducible subspaces (according to the representation theory).

More precisely, the subspaces $\mathscr{T}_{1}(V), \mathscr{W}_{4}^{\prime}$ and $\mathscr{W}_{4}^{\prime \prime}$ are isomorphic to each other and $\mathscr{W}_{3}^{\prime}$ is isomorphic to $\mathscr{W}_{3}^{\prime \prime}$. It can be seen that these isomorphisms commute with the induced representation of $U(n)$.

This implies that there is an infinite number of invariant subspaces of $\mathscr{T}(V)$. The choice among the different decompositions is due to the geometrical aspects of the problem which is studied.

In the next theorem we give the explicit definitions of the eight invariant subspaces of Theorem 4.3.

Theorem 4.4. If $\operatorname{dim} V=2 n$, we have

$$
\begin{gathered}
\mathscr{T}_{1}(V)=\left\{T \in \mathscr{T}(V) / T_{X Y Z}=\langle X, Y\rangle \phi(Z)-\langle X, Z\rangle \phi(Y), \phi \in V^{*}\right\}, \\
\operatorname{Ker} \psi_{2}=\left\{T \in \mathscr{T}(V) / T_{X Y Z}=T_{X J Y J Z},\left(\mathfrak{F}_{X Y Z} T_{X Y Z}=0, c_{12}(T)=0\right\},\right. \\
\mathscr{W}_{2}^{\prime}=\left\{T \in \mathscr{T}(V) / \mathfrak{F}_{X Y Z} T_{X Y Z}=0, T_{X Y Z}=-\frac{1}{3}\left(T_{J X J Y Z}+T_{J X Y J Z}+T_{X J Y J Z}\right)\right\}, \\
\mathscr{W}_{3}^{\prime}=\left\{T \in \mathscr{T}(V) / T_{X Y Z}=T_{J X J Y Z}+T_{J X Y J Z}+T_{X J Y J Z}\right. \\
\left.=-2 T_{X J Y J Z}+T_{Y J Z J X}+T_{Z J X J Y}, c_{12}(T)=0\right\}, \\
\mathscr{W}_{4}^{\prime}=\left\{T \in \mathscr{T}(V) / T_{X Y Z}=\langle X, Y\rangle v(Z)-\langle X, Z\rangle v(Y)+\frac{2 n-1}{3}(2\langle Y, J Z\rangle v(J X)\right. \\
\left.-\langle X, J Y\rangle v(J Z)-\langle J X, Z\rangle v(J Y)), v \in V^{*}\right\}, \\
\mathscr{W}_{1}^{\prime \prime}=\left\{T \in \mathscr{T}(V) / T_{X Y Z}=-T_{Y X Z}=-T_{J X J Y Z}\right\},
\end{gathered}
$$




$$
\begin{gathered}
\mathscr{W}_{3}^{\prime \prime}=\left\{T \in \mathscr{T}(V) / T_{X Y Z}=-T_{Y X Z}=T_{X J Y J Z}+T_{J X J Y Z}+T_{J X Y J Z}, \bar{c}_{12}(T)=0\right\}, \\
\mathscr{W}_{4}^{\prime \prime}=\left\{T \in \mathscr{T}(V) / T_{X Y Z}=\langle J X, Y\rangle \xi(Z)+\langle J Z, X\rangle \xi(Y)+\langle J Y, Z\rangle \xi(X), \xi \in V^{*}\right\},
\end{gathered}
$$

where $X, Y, Z \in V, \mathfrak{G}_{X Y Z}$ denotes the cyclic sum with respect to $X, Y, Z$ and

$$
c_{12}(T)(X)=\sum_{i=1}^{2 n} T_{e_{i} e_{i} X}, \quad \bar{c}_{12}(T)(X)=\sum_{i=1}^{2 n} T_{e_{i} J e_{i} X}, \quad X \in V,
$$

$\left(e_{1}, \ldots, e_{2 n}\right)$ is an orthonormal basis of $V$.

Proof. The defining conditions for $\mathscr{W}_{1}^{\prime \prime}, \mathscr{W}_{3}^{\prime \prime}, \mathscr{W}_{4}^{\prime \prime}$ can be easily obtained using the isomorphism $\psi_{3}$ (see Theorem 4.1). We need only to consider the subspace $\mathscr{T}_{2}(V)$.

First of all, let us define

$$
\mathscr{T}_{2}^{0}(V)=\left\{T \in \mathscr{T}(V) / \bar{c}_{12}(T)=0\right\} .
$$

It is clear that $\mathscr{T}_{2}^{0}(V)$ is an invariant subspace of $\mathscr{T}_{2}(V)$ and

$$
\mathscr{T}_{2}(V)=\mathscr{T}_{2}^{0}(V) \oplus \mathscr{T}_{2}^{0}(V)^{\perp}
$$

Obviously, $\mathscr{T}_{2}^{0}(V)^{\perp}$ coincides with $\mathscr{W}_{4}^{\prime}$, and its explicit expression is a matter of computation.

Next, we introduce the endomorphism $\xi$ of $\mathscr{T}_{2}^{0}(V)$ given by

$$
(\xi)_{X Y Z}=\frac{1}{4}\left(T_{X Y Z}-T_{J X J Y Z}-T_{J X Y J Z}-T_{X J Y J Z}\right), \quad X, Y, Z \in V .
$$

It is easy to check that

(i) $\xi$ commutes with the action of $U(n)$,

(ii) $\xi^{2}=\xi$,

(iii) $\psi_{2}: \operatorname{Im} \xi \rightarrow \mathscr{W}_{2}$ is an isomorphism,

(iv) $\mathscr{T}_{2}^{0}=\operatorname{Ker} \xi \oplus \operatorname{Im} \xi$ and $\operatorname{Ker} \xi=\operatorname{Ker} \psi_{2} \oplus \mathscr{W}_{3}^{\prime}, \operatorname{Im} \xi=\mathscr{W}_{2}^{\prime}$.

By (iv) we have the explicit description of $\mathscr{W}_{2}^{\prime}$. Moreover the endomorphism $\chi$ of $\operatorname{Ker} \xi$ given by

$$
\chi(T)_{X Y Z}=\frac{1}{4}\left(3 T_{X Y Z}-2 T_{X J Y J Z}+T_{Y J Z J X}+T_{Z J X J Y}\right), \quad X, Y, Z \in V,
$$

has the following properties

(i) $\chi$ commutes with the action of $U(n)$,

(ii) $\chi^{2}=\chi$,

(iii) $\operatorname{Ker} \xi=\operatorname{Ker} \chi \oplus \operatorname{Im} \chi$ and $\operatorname{Ker} \chi=\operatorname{Ker} \psi_{2}, \operatorname{Im} \chi=\mathscr{W}_{3}^{\prime}$.

These complete the proof of the theorem. 
By explicit calculations, one gets

$$
\begin{gathered}
\phi(X)=\frac{1}{2 n-1} c_{12}(T)(X), \\
v(X)=\frac{3}{4\left(1-n^{2}\right)} \bar{c}_{12}(T)(J X), \\
\xi(X)=\frac{1}{2(n-1)} \bar{c}_{12}(T)(X), \quad X \in V .
\end{gathered}
$$

\section{Geometric results}

Let $(M, g, J)$ be a homogeneous almost Hermitian manifold of dimension $2 n$. For every $p \in M,\left(T_{p} M, g_{p}, J_{p}\right)$ is a Hermitian vector space. Given an orthonormal basis $\left(e_{1}, \ldots, e_{n}, J e_{1}, \ldots, J e_{n}\right)$ of $T_{p} M$, there is a standard representation of $U(n)$ on $T_{p} M$. Hence it is possible to decompose the vector space $\mathscr{T}\left(T_{p} M\right)$ as in section 2 or 4. Let $\mathscr{H}$ denote a subspace of $\mathscr{T}\left(T_{p} M\right)$ invariant with respect to the representation of $U(n)$.

We say that $M$ is of type $\mathscr{H}$ if $T_{p} \in \mathscr{H}$, for all $p \in M$, where $T$ is the corresponding homogeneous almost Hermitian structure (see Theorem 1.2). Then we simply write: $M \in \mathscr{H}$.

We deduce now some geometrical consequences from the decomposition given in Theorem 4.4. In [16], Tricerri and Vanhecke proved the following theorem.

Theorem 5.1. Let $(M, g)$ be a connected, complete and simply connected Riemannian manifold. Then $(M, g)$ admits a nonvanishing homogeneous (Riemannian) structure $T \in \mathscr{T}_{1}$ if and only if $(M, g)$ is isometric to the hyperbolic space.

Let $H^{2 n}=\left\{\left(y^{1}, \ldots, y^{2 n}\right) \in \mathbb{R}^{2 n} \mid y^{1}>0\right\}$ be the hyperbolic space of dimension $2 n$ with the metric

$$
d s^{2}=\left(c y^{1}\right)^{-2} \sum_{i=1}^{2 n}\left(d y^{i}\right)^{2}, \quad c \in \mathbb{R}, \quad c>0
$$

The tensor $T$ given by

$$
T_{X} Y=g(X, Y)-g(\zeta, Y) X, \quad X, Y \in \mathfrak{X}\left(H^{2 n}\right),
$$

where $g$ denotes the Riemannian metric on $H^{2 n}$ and $\zeta=c^{2} y^{1}\left(\partial / \partial y^{1}\right)$, is a (Riemannian) homogeneous structure.

If $J$ is an almost complex structure on $H^{2 n}$, let us put

$$
J\left(\frac{\partial}{\partial y^{h}}\right)=\sum_{k=1}^{2 n} J_{h}^{k}\left(\frac{\partial}{\partial y^{k}}\right), \quad J_{h}^{k} \in \mathscr{C}^{\infty}\left(H^{2 n}\right), \quad h=1, \ldots, 2 n .
$$


An easy computation shows that $J$ satisfies the condition $\tilde{\nabla} J=0$ (see Theorem 1.2) if and only if the components $J_{h}^{k}$ are constant on $H^{2 n}$. Hence it is possible to find a coordinate system, obtained from the previous one by a linear combination with constant coefficients, such that $J$ has the same expression as the standard complex structure of $\mathbb{C}^{n}$. So we have

Theorem 5.2. Let $(M, \tilde{g}, \tilde{J})$ be a connected, complete and simply connected almost Hermitian manifold of dimension $2 n>2 . M$ admits a nonvanishing almost Hermitian homogeneous structure of type $\mathscr{T}_{1}$ if and only if there is a holomorphic isometry from $(M, \tilde{g}, \widetilde{J})$ onto $\left(H^{2 n}, g, J\right)$.

Moreover $M$ is globally conformal Kähler but not Kähler.

About the last remark, we recall that $M$ is a locally conformal Kähler manifold if there exists a globally defined 1-form $\omega$ satisfying

$$
d F=\omega \wedge F \text { and } d \omega=0,
$$

where $F$ is the Kähler, form of $M$; $\omega$ is called the Lee form.

If $\omega$ is also exact, $M$ is a globally conformal Kähler manifold and this happens, for example, if $M$ is simply connected.

If $\operatorname{dim} M>4$, in [9] it is shown that $M$ is a locally conformal Kähler manifold if and only if $\nabla F \in \mathscr{W}_{4}$.

Finally, we remark that the homogeneous structure $T$ corresponds to the representation of $H^{2 n}$ as a solvable Lie group which is the semidirect product of the multiplicative group $\mathbb{R}_{0}^{+}=\{x \in \mathbb{R} \mid x>0\}$ and the additive group $\mathbb{R}^{2 n-1}$ with the product: $\left(x^{1}, \ldots, x^{2 n}\right)\left(y^{1}, \ldots, y^{2 n}\right)=\left(x^{1} y^{1}, x^{1} y^{2}+x^{2}, \ldots, x^{1} y^{2 n}+x^{2 n}\right)$. The complex structure $J$ considered above is left invariant but $H^{2 n}$ is not a complex Lie group (see Theorem 2.3 of [2]).

Theorem 5.3. The connected, simply connected, almost Hermitian naturally reductive homogeneous manifolds of dimension $2 n \geqq 6$ are classified into eight classes, given by all the invariant subspaces of the decomposition

$$
\mathscr{T}_{3}=\mathscr{W}_{1}^{\prime \prime} \oplus \mathscr{W}_{3}^{\prime \prime} \oplus \mathscr{W}_{4}^{\prime \prime}
$$

Proof. This follows from the Theorem 4.1 and from the fact that a homogeneous Riemannian manifold $M$ belongs to the class $\mathscr{T}_{3}$ if and only if it is naturally reductive (see [16, Chapter 6]).

From Remark (b) of Theorem 4.3, in this case, there are no equivalent components and we obtain a complete classification.

In the case of $\operatorname{dim} M=4$, we get $\mathscr{T}_{3}=\mathscr{W}_{4}^{\prime \prime}=\psi_{3}^{-1}\left(\mathscr{W}_{4}\right)$. Gray and Hervella [9] proved that if $\operatorname{dim} M=4, \mathscr{W}_{4}$ is the class of Hermitian manifold (i.e. the Nijenhuis tensor of $M$ vanishes). So, it follows that: 
Corollary 5.4. A four dimensional almost Hermitian, naturally reductive, homogeneous manifold is a Hermitian manifold.

From the classification of the almost Hermitian manifolds, [9], we get:

Theorem 5.5. Let $M$ be a connected, simply connected almost Hermitian homogeneous manifold of dimension $2 n \geqq 6$. Then

(a) $M \in \mathscr{W}_{1}^{\prime \prime}$ if and only if $M$ is a naturally reductive nearly Kähler manifold.

(b) $M \in \mathscr{W}_{4}^{\prime \prime}$ if and only if $M$ is a naturally reductive locally conformal Kähler manifold.

(c) $M \in \mathscr{W}_{3}^{\prime \prime} \oplus \mathscr{W}_{4}^{\prime \prime}$ if and only if $M$ is a naturally reductive Hermitian manifold.

Proof. By definition, $M$ is a nearly Kähler manifold if $\nabla_{X}(F)(X, Y)=0, X, Y \in \mathfrak{X}(M)$, and $F$ is the Kähler form on $M$. In [9] it is shown that $M$ is a nearly Kähler manifold if and only if $\nabla F \in \mathscr{W}_{1}$. (b) and (c) follow from the observations concerning Theorem 5.2 and Corollary 5.4 .

Theorem 5.6. The almost Hermitian homogeneous structures of type $\mathscr{T}_{2}$ are classified into sixteen classes, given by all the invariant subspaces of the decomposition

$$
\mathscr{T}_{2}=\operatorname{Ker} \psi_{2} \oplus \mathscr{W}_{2}^{\prime} \oplus \mathscr{W}_{3}^{\prime} \oplus \mathscr{W}_{4}^{\prime}
$$

Proof. It follows directly from Theorem 4.2.

For the almost Kähler manifold it is possible to deduce a nice property.

Theorem 5.7. An almost Kähler, naturally reductive, homogeneous manifold is locally Hermitian symmetric.

Proof. $M$ is an almost Kähler manifold if its Kähler form is closed. Gray and Hervella [9] proved that every almost Kähler manifold belongs to the class $\mathscr{W}_{2}$. From the previous theorems it follows that an almost Hermitian homogeneous structure $T \in \mathscr{T}_{3}$ on an almost Kähler homogeneous manifold vanishes. Then the manifold is locally Hermitian symmetric (see [10] and [16]).

Remark. Theorem 5.7 provides a generalization and a direct verification of a result of Deloff [6] concerning Kähler manifolds.

Theorem 5.8. A homogeneous almost Hermitian manifold is Kählerian if and only if it belongs to the subspace $\mathscr{T}_{+}$defined in Theorem 2.1.

Proof. The covariant derivative of the Kähler form of a Kähler manifold vanishes. Then, from condition (iv) of Theorem 1.2 one gets

$$
T_{X J Y Z}+T_{X Y J Z}=0, \quad X, Y, Z \in \mathfrak{X}(M),
$$

where $T$ is an almost Hermitian homogeneous structure. But this is precisely the definition of $\mathscr{T}_{+}$. 
Remarks. (a) Let $M$ be a connected homogeneous almost Hermitian manifold of dimension $2 n \geqq 6$. Then $M \in \mathscr{H}_{5}$ if and only if $M$ is a naturally reductive nearly Kähler manifold. This follows from Theorem 5.5(a) and from the definition of $\mathscr{H}_{5}$ and $\mathscr{W}_{1}^{\prime \prime}$.

(b) If $\operatorname{dim} V=2$, then $\mathscr{T}(V)=\mathscr{H}_{4}$ (see Theorem 2.3) and $T \in \mathscr{H}_{4}$ can be written as follows

$$
T_{X} Y=\langle X, Y\rangle \zeta-\langle Y, \zeta\rangle X-\langle X, J Y\rangle J \zeta+\langle J Y, \zeta\rangle J X+2\langle J X, \zeta\rangle J Y, \quad X, Y \in V,
$$

where $\zeta \in V$ is defined by $\langle\zeta, X\rangle=\psi_{2}(X), X \in V$.

By means of the identity

$$
\|\zeta\|^{2} X=\langle X, \zeta\rangle \zeta+\langle X, J \zeta\rangle \zeta, \quad X \in V
$$

it is easy to show that

$$
T_{X} Y=4\{\langle X, Y\rangle \zeta-\langle Y, \zeta\rangle X\}, \quad X, Y \in V .
$$

Hence, the class $\mathscr{H}_{4}$ coincides with $\mathscr{T}_{1}(V)$ and we may deduce the consequences of Theorem 5.2.

\section{Examples}

(a) Let $(M, g)$ be a 3-symmetric Riemannian space, i.e. for each $p \in M$ there exists an isometry $\theta_{p}$ of $M$ such that

(i) $p$ is an isolated fixed point of $\theta_{p}$,

(ii) $\left(\theta_{p}^{3}\right)_{*}=I$, where $I$ denotes the identity isomorphism of $T_{p} M$.

It is well known that a 3-symmetric space can be endowed with an almost complex structure $J$ which is compatible with metric $g$ [8]. Hence, for every $p \in M, \theta_{p}$ becomes a holomorphic isometry with respect to $J$. In particular, $(M, g, J)$ satisfies the conditions

$$
\left(\nabla_{X} J\right)(Y)+\left(\nabla_{J X} J\right)(J Y)=0, \quad X, Y \in \mathfrak{X}(M),
$$

that is: $M$ belongs to the class $\mathscr{W}_{1} \oplus \mathscr{W}_{2}$ of the classification of almost Hermitian manifolds [9]; further, the tensor field $T$ given by

$$
T_{X} Y=\frac{1}{2} J\left(\nabla_{X} J\right)(Y), \quad X, Y \in \mathfrak{X}(M),
$$

is an almost Hermitian homogeneous structure on $M$. Tricerri and Vanhecke [16] proved also that $T \in \mathscr{T}_{2} \oplus \mathscr{T}_{3}$, and $T \in \mathscr{T}_{3}$ if and only if $(M, g, J)$ is a nearly Kähler 3-symmetric space. Moreover, $T \in \mathscr{T}_{2}$ if and only if $(M, g, J)$ is an almost Kähler 3-symmetric manifold. The same results are easily deduced from Theorems 4.1 and 4.2. In fact, nearly Kähler manifolds and almost Kähler manifolds belong, respectively, to the classes $\mathscr{W}_{1}$ and $\mathscr{W}_{2}$, as has been observed.

(b) The following example shows that the inclusion between $\mathscr{H}_{2} \oplus \mathscr{H}_{3} \oplus \mathscr{H}_{4}$ and $\mathscr{H}_{2} \oplus \mathscr{H}_{3} \oplus \mathscr{H}_{4} \oplus \mathscr{H}_{6}$ is strict. Let us consider the 4-dimensional Lie group $G$ given by 


$$
G=\left\{\left(\begin{array}{cccc}
1 & x & z & 0 \\
0 & 1 & y & 0 \\
0 & 0 & 1 & 0 \\
0 & 0 & 0 & e^{2 \pi i t}
\end{array}\right), x, y, z, t \in \mathbb{R}\right\}
$$

Endowed with the left invariant metric

$$
d s^{2}=d x^{2}+d y^{2}+(d z-x d y)^{2}+d t^{2},
$$

$G$ becomes a Riemannian homogeneous manifold, diffeomorphic to $\mathbb{R}^{3} \times S^{1}$. In fact $G$ can be considered as the direct product of the Heisenberg group and the circle. An orthonormal basis of the Lie algebra $\mathfrak{g}$ of $G$ is given by

$$
e_{1}=\frac{\partial}{\partial x}, \quad e_{2}=\frac{\partial}{\partial y}+\frac{\partial}{\partial z}, \quad e_{3}=\frac{\partial}{\partial z}, \quad e_{4}=\frac{\partial}{\partial t} .
$$

From the Cartan structural equations, we deduce the connection 1-forms for the Levi Civita connection of $g$

$$
\omega_{12}=-\frac{1}{2}(d z-x d y), \quad \omega_{13}=-\frac{1}{2} d y, \quad \omega_{23}=\frac{1}{2} d x, \quad \omega_{14}=\omega_{24}=\omega_{34}=0 .
$$

Hence the nonzero components of the Riemannian curvature tensor are:

$$
R_{e_{1} e_{2} e_{1} e_{2}}=-\frac{3}{4}, \quad R_{e_{2} e_{3} e_{2} e_{3}}=\frac{1}{4}, \quad R_{e_{1} e_{3} e_{1} e_{3}}=\frac{1}{4} .
$$

Let us define an almost complex structure $J$ on $G$ as follows:

$$
J e_{1}=-e_{4}, \quad J e_{2}=e_{3} .
$$

$(G, g, J)$ is an almost Kähler homogeneous manifold because the Kähler form of $G$

$$
F=d t \wedge d x+d y \wedge(d z-x d y)
$$

is closed; but $(G, g, J)$ is not a Kähler manifold (for detailed computations, see [1]). By solving explicitly the Ambrose-Singer-Sekigawa equations, one can find that on $G$ there is only one almost Hermitian homogeneous structure $T$ given by:

$$
\begin{gathered}
T_{e_{1}} e_{2}=\frac{1}{2} e_{3}, \quad T_{e_{1}} e_{3}=-\frac{1}{2} e_{2}, \quad T_{e_{2}} e_{3}=\frac{1}{2} e_{1}, \quad T_{e_{2}} e_{1}=-\frac{1}{2} e_{3}, \\
T_{e_{3}} e_{1}=-\frac{1}{2} e_{2}, \quad T_{e_{3}} e_{2}=\frac{1}{2} e_{1}, \quad T_{e_{1}} e_{j}=0 \text { otherwise. }
\end{gathered}
$$

By a direct calculation, it is possible to find the class which $T$ belongs to: $T \in \mathscr{H}_{2} \oplus \mathscr{H}_{3} \oplus \mathscr{H}_{4} \oplus \mathscr{H}_{6}$. From Theorem 5.8, we have: $T \notin \mathscr{H}_{2} \oplus \mathscr{H}_{3} \oplus \mathscr{H}_{4}$. If we consider the second decomposition, we find that: $T \in \mathscr{W}_{2}^{\prime}$. 
In [1] the compact manifold $M=G / \Gamma$ is studied, where $G$ is the above Lie group and $\Gamma$ is the discrete subgroup of $G$ generated by the matrices of $G$ whose entries are integers. $M$ is the Thurston example [14] of a compact symplectic manifold admitting no Kähler structure. On the other hand, according to Theorem 4.10 of [20], if $M=G / K$ is a symplectic homogeneous manifold and $G$ is a nilpotent Lie group, $G$ must be abelian. Then the manifold $M=G / \Gamma$ and its generalizations ([4 and 5]) are not almost Kähler homogeneous manifolds because they are not symplectic homogeneous manifolds. However, they are examples of locally homogeneous almost Kähler manifolds which are not homogeneous, [15].

(c) We describe now a family of examples of homogeneous almost Hermitian manifolds of class $\mathscr{T}_{2}$ (see [11] for the computations). Let us consider the Lie group

$$
G=\left\{\left(\begin{array}{cccc}
e^{\alpha t} & 0 & 0 & x \\
0 & e^{\beta t} & 0 & y \\
0 & e^{-(\alpha+\beta) r} & 0 & z \\
0 & 0 & 0 & 1
\end{array}\right), x, y, z, t \in \mathbb{R}, \alpha, \beta \neq 0, \alpha, \beta \in \mathbb{R}, \text { constant }\right\},
$$

with the metrics:

$$
d s^{2}=e^{-2 \alpha t} d x^{2}+e^{-2 \beta t} d y^{2}+e^{2(\alpha+\beta) t} d z^{2}+\left(d t-u e^{(\alpha+\beta) t} d z\right)^{2}
$$

$\alpha, \beta, u \in \mathbb{R} ; \alpha, \beta \neq 0$.

An orthonormal frame field on $G$ is given by

$$
e_{1}=\frac{\partial}{\partial t}, \quad e_{2}=e^{\alpha t} \frac{\partial}{\partial x}, \quad e_{3}=e^{\beta t} \frac{\partial}{\partial y}, \quad e_{4}=e^{-(\alpha+\beta) t} \frac{\partial}{\partial z}+u \frac{\partial}{\partial t} .
$$

In general, if $G$ is a Lie group with a left invariant metric $g$, the tensor $T$ is given by

$$
2 g\left(T_{X} Y, Z\right)=g([X, Y], Z)-g([Y, Z], X)+g([Z, X], Y), \quad X, Y, Z \in \mathfrak{g},
$$

where $g$ denotes the Lie algebra of $G$.

Let $\nabla$ the Levi Civita connection on $G$, the metric connection $\tilde{\nabla}=\nabla-T$ satisfies: $\tilde{\nabla}_{X} Y=0$, for all $X, Y \in \mathfrak{g}$. Hence, the curvature tensor $\tilde{R}$ of $\tilde{\nabla}$ vanishes and $T$ is an homogeneous structure on $G,[16]$.

In our case, $T$ is given by

$$
\begin{gathered}
T_{e_{2}} e_{1}=\alpha e_{2}, \quad T_{e_{3}} e_{1}=\beta e_{3}, \quad T_{e_{4}} e_{1}=-(\alpha+\beta) e_{4}, \quad T_{e_{2}} e_{2}=-\alpha e_{1}, \\
T_{e_{3}} e_{3}=-\beta e_{1}, \quad T_{e_{4}} e_{4}=(\alpha+\beta) e_{1}, \quad T_{e_{1}} e_{j}=0 \text { otherwise. }
\end{gathered}
$$

Let $J$ be any left invariant almost complex structure on $G$, compatible with $d s^{2}$. It is clear that, by the definition of $\tilde{\nabla}$, the fourth condition of Theorem 1.2 is always satisfied. It follows that $\left(G, d s^{2}, J\right)$ is an almost Hermitian homogeneous manifold. It can be checked that $G$ belongs to the class $\mathscr{T}_{2}$. In fact, it is the unique irreducible four dimensional manifold of type $\mathscr{T}_{2}$ which is not a 3-symmetric space [11]. 
Computing the trace $\bar{c}_{12}(T)(X)=\sum_{i=1}^{4} T_{e_{i} J e_{i} X}, X \in \mathrm{g}$, one realizes that $\bar{c}_{12}(T)=0$ if and only if $\alpha+\beta=0$.

Hence, if $\alpha+\beta=0, G$ belongs to $\operatorname{Ker} \psi_{2} \oplus \mathscr{W}_{2}^{\prime} \oplus \mathscr{W}_{3}^{\prime}$ but $G$ does not belong to $\operatorname{Ker} \psi_{2} \cup \mathscr{W}_{2}^{\prime} \cup \mathscr{W}_{3}^{\prime}$.

If $\alpha+\beta \neq 0, G$ does not belong to $\operatorname{Ker} \psi_{2} \cup \mathscr{W}_{2}^{\prime} \cup \mathscr{W}_{3}^{\prime} \cup \mathscr{W}_{4}^{\prime}$.

Acknowledgements. We wish to thank F. Tricerri for the choice of the subject, for his encouragement and several useful discussions. Also thanks are due to A. Gray and S. Salamon. Moreover the authors wish to thank the referee for his helpful suggestions.

\section{REFERENCES}

1. E. AgBenA, An example of an almost Kähler manifold which is not Kählerian, Boll. Un. Mat. Ital. (6) (1984), 383-392.

2. E. Abbena and A. Grassi, Hermitian left invariant metrics on complex Lie groups and cosymplectic Hermitian manifolds, Boll. Un. Mat. Ital. (6), 5-A (1986), 371-379.

3. W. Ambrose and I. M. Singer, On homogeneous Riemannian manifolds, Duke Math. J. 25 (1958), 647-669.

4. L. A. Cordero, M. Fernandez and M. De Leon, Examples of compact non-Kähler almost Kähler manifolds, Proc. Amer. Math. Soc. 95 (1985), 280-286.

5. L. A. Cordero, M. Fernandez and A. Gray, Symplectic manifolds with no Kähler structures, Topology 25 (1986), 375-380.

6. E. D. Deloff, Naturally reductive metrics and metrics with volume preserving geodesic symmetries on NC algebras (Ph.D. Thesis, The State University of New Jersey, 1979).

7. A. Gray, Nearly Kähler manifolds, J. Differential Geom. 4 (1970), 283-309.

8. A. GRAY, Riemannian manifolds with geodesic symmetries of order 3, J. Differential Geom. 7 (1972), 343-369.

9. A. Gray and L. M. Hervella, The sixteen classes of almost Hermitian manifolds, Ann. Mat. Pura Appl. 123 (1980), 35-58.

10. S. Kobayashi and K. Nomizu, Foundations of differential geometry, Vol. I, II (Interscience Publ., New York, 1969).

11. O. Kowalski and F. TricerRI, Riemannian manifolds of dimension $\leqq 4$ admitting a homogeneous structure of type $\mathscr{T}_{2}$, preprint.

12. N. IwahorI, Some remarks on tensors invariants of $O(n), U(n), S p(n), J$. Math. Soc. Japan 10 (1958), 143-160.

13. K. Sekigawa, Notes on homogeneous almost Hermitian manifolds, Hokkaido Math. J. 7 (1978), 206-213.

14. W. P. Thurston, Some simple examples of symplectic manifolds, Proc. Amer. Math. Soc. 55 (1976), 467-468.

15. F. Tricerri, Varietà Riemanniane omogenee, Atti del Convegno Nazionale del GNSAGA del CNR, Torino 4-6 Ottobre 1984, 221-245.

16. F. Tricerri and L. VANhecke, Homogeneous structures on Riemannian manifolds (London Math. Soc. Lect. Notes 83, Cambridge Univ. Press, 1983). 
17. F. Tricerri and L. Vanhecke, Special homogeneous structures on Riemannian manifolds, Proc. Colloq. Diff. Geom., Hajduszoboszlo (Debrecen, 1984), to appear.

18. F. Tricerri and L. Vanhecke, Naturally reductive homogeneous spaces and Heisenberg groups, Compositio Math. 52 (1984), 389-408.

19. F. Tricerri and L. Vanhecke, Curvature tensors on almost Hermitian manifolds, Trans. Amer. Math. Soc. 267 (1981), 365-398.

20. P. B. Zwart and W. M. Boothby, On compact homogeneous symplectic manifolds, Ann. Inst. Fourier (Grenoble) 30 (1980), 129-157.

Dipartimento di Matematica

UNIVERSITÁ DI TORINO

via Principe Amedeo, 8

10123 TORINO

ITALY 